\title{
Is there an association between early cognitive decline after cardiac surgery and postoperatively elevated cortisol?: A prospective cohort study
}

\author{
Glumac S ${ }^{1}$, Kardum $\mathrm{G}^{2}$ and Karanović $\mathrm{N}^{1}$ \\ ${ }^{1}$ Department of Anaesthesiology and Intensive Care, University Hospital of Split, Croatia \\ ${ }^{2}$ Department of Psychology, Faculty of Humanities and Social Sciences, University of Split, Croatia
}

Background and Goal of Study: Cognition is impaired by the sustained elevation of cortisol levels; ${ }^{1}$ therefore, the prolonged and pronounced stress response to a surgical procedure $^{2}$ may play an important role in the development of postoperative cognitive decline (POCD). A recent study reported that patients with higher cortisol levels on the 1st postoperative morning after cardiac surgery exhibited an increased risk of early POCD. ${ }^{3}$ To gain further insight into the stress response to a surgical procedure as a potential risk factor for early POCD, we measured the perioperative cortisol levels at multiple time points. We hypothesized that higher postoperative cortisol levels are related to the occurrence of early POCD after cardiac surgery.

Material and Methods: This prospective cohort study enrolled 125 patients undergoing elective cardiac surgery with or without cardiopulmonary bypass (CPB). Patient serum cortisol levels were determined one day before surgery (at 08:00) and on the 1st (at 08:00, 16:00 and 24:00), 3rd (at 08:00) and 5th (at 08:00) postoperative days. A battery of six neuropsychological tests was used to assess the participants two days before the surgical procedure and on the 6th postoperative day. POCD was defined as a decrease in performance of 1 SD or greater between the postoperative and preoperative $z$ scores on at least one neuropsychological test. A mixed-design ANOVA was used to determine the correlations of the perioperative cortisol levels with the occurrence of POCD and with the surgical technique performed.

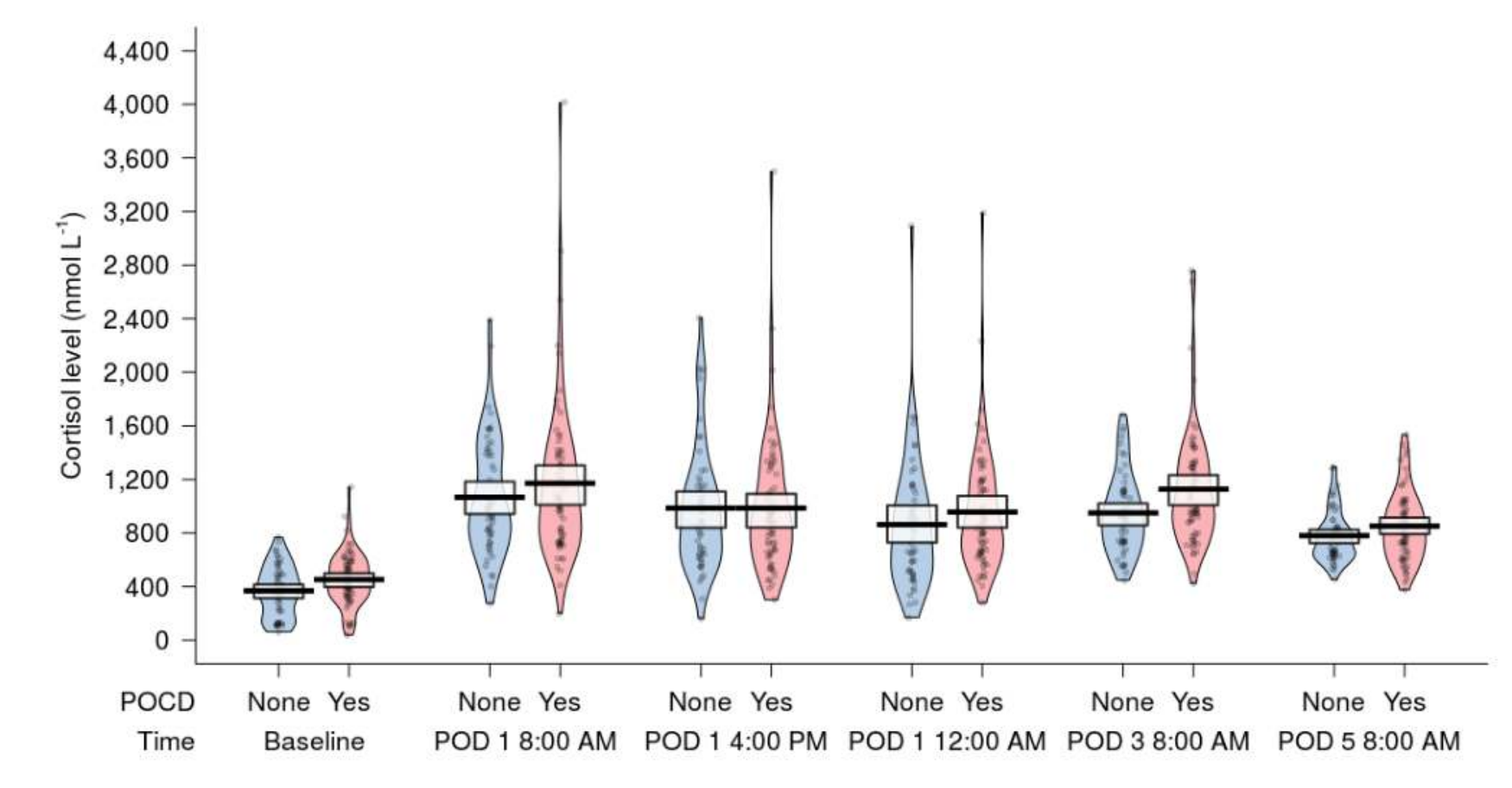

Results: Mixed-design ANOVA showed no statistically significant differences in the cortisol levels between non-POCD and POCD patients ( $F=0.52, P=0.690$, Fig. 1 ) or between patients with and without $\mathrm{CPB}(\mathrm{F}=2.02, \mathrm{P}=$ 0.103 , Fig. 2) at the six perioperative time points. Therefore, this study shed new light, revealing that the high postoperative cortisol levels merely reflect the stress response to the surgical procedure, while the real cause of POCD after cardiac surgery involves another mechanism. Additionally, we showed that cardiac manipulation during beating-heart surgery may lead to significant haemodynamic impairment reflected by a pronounced cortisol response, which therefore negates the benefits of avoiding CPB in terms of the stress hormone response.

Conclusion: The occurrence of early POCD and the use of CPB were not associated with significantly higher cortisol levels in this study with a repeated measurement design.

\section{References}

1. Lupien SJ, Maheu F, Tu M, Fiocco A, Schramek TE. The effects of stress and stress hormones on human cognition: Implications for the field of brain and cognition. Brain Cogn 2007; 65:209-237.

2. Gibbison B, Spiga F, Walker JJ, et al. Dynamic pituitary-adrenal interactions in response to cardiac surgery. Crit Care Med 2015; 43:791-800. 3. Mu DL, Li LH, Wang DX, et al. High postoperative serum cortisol level is associated with increased risk of cognitive dysfunction early after coronary artery bypass graft surgery: a prospective cohort study. PLoS One 2013; 8:e77637. 